\title{
Head Direction Cell Representations Maintain Internal Coherence during Conflicting Proximal and Distal Cue Rotations: Comparison with Hippocampal Place Cells
}

\author{
D. Yoganarasimha, Xintian Yu, and James J. Knierim \\ Department of Neurobiology and Anatomy, W. M. Keck Center for the Neurobiology of Learning and Memory, The University of Texas Medical School at \\ Houston, Houston, Texas 77225
}

\begin{abstract}
Place cells of the hippocampal formation encode a spatial representation of the environment, and the orientation of this representation is apparently governed by the head direction cell system. The representation of a well explored environment by CA1 place cells can be split when there is conflicting information from salient proximal and distal cues, because some place fields rotate to follow the distal cues, whereas others rotate to follow the proximal cues (Knierim, 2002a). In contrast, the CA3 representation is more coherent than CA1, because the place fields in CA3 tend to rotate in the same direction (Lee et al., 2004). The present study tests whether the head direction cell network produces a split representation or remains coherent under these conditions by simultaneously recording both CA1 place cells and head direction cells from the thalamus. In agreement with previous studies, split representations of the environment were observed in ensembles of CA1 place cells in $\sim 75 \%$ of the mismatch sessions, in which some fields followed the counterclockwise rotation of proximal cues and other fields followed the clockwise rotation of distal cues. However, of 225 recording sessions, there was not a single instance of the head direction cell ensembles revealing a split representation of head direction. Instead, in most of the mismatch sessions, the head direction cell tuning curves rotated as an ensemble clockwise (94\%) and in a few sessions rotated counterclockwise (6\%). The findings support the notion that the head direction cells may be part of an attractor network bound more strongly to distal landmarks than proximal landmarks, even under conditions in which the CA1 place representation loses its coherence.
\end{abstract}

Key words: place cells; head direction cells; ensemble recording; attractor neural network; spatial orientation; single units

\section{Introduction}

The hippocampus is a critical structure involved in navigation, context-dependent learning, and episodic memory (O'Keefe and Nadel, 1978; Vargha-Khadem et al., 1997; Tulving and Markowitsch, 1998; Eichenbaum 2004). The most salient correlate of hippocampal principal cell activity in the behaving rat is the spatial location of the rat (O'Keefe and Dostrovsky, 1971; Muller et al., 1987). These place cells are controlled by a complex interaction between self-motion cues (Sharp et al., 1995; Wiener et al., 1995; McNaughton et al., 1996; Knierim, 2002b; Stackman et al., 2002) and salient landmarks in the environment (O'Keefe and Conway, 1978; Muller and Kubie, 1987; Young et al., 1994; Save and Poucet, 2000; Brown and Skaggs, 2002; Knierim, 2002a). When the distal and proximal landmarks are rotated in opposite directions, the CA1 representation can split in two, because some place fields rotate to follow the proximal landmarks and others rotate

Received Sept. 13, 2005; revised Nov. 10, 2005; accepted Nov. 11, 2005.

This work was supported by United States Public Health Service Grants R01 NS39456 and K02 MH63297 and by the Lucille P. Markey Charitable Trust. We thank Geeta Rao and Inah Lee for their help in data collection and analysis and Jennifer Siegel for comments on this manuscript.

Correspondence should be addressed to Dr. James J. Knierim, Department of Neurobiology and Anatomy, The University of Texas Medical School at Houston, P.0. Box 20708, Houston, TX 77225. E-mail: james.j.knierim@uth.tmc.edu.

DOI:10.1523/JNEUROSCI.3885-05.2006

Copyright $\odot 2006$ Society for Neuroscience $\quad$ 0270-6474/06/260622-10\$15.00/0 to follow the distal landmarks (Shapiro et al., 1997; Tanila et al., 1997; Zinyuk et al., 2000; Knierim, 2002a). In contrast, the spatial representation in CA3 maintains a greater degree of coherence, because many more place fields rotate as an ensemble with one set of landmarks dominating over the other, perhaps reflecting the pattern completion/autoassociative network properties hypothesized to underlie the computational functions of the CA3 recurrent collateral system (Guzowski et al., 2004; Lee et al., 2004).

The orientation of the hippocampal spatial representation relative to the external environment is thought to be derived from the head direction cell system (McNaughton et al., 1996; Muller et al., 1996; O'Keefe and Burgess, 1996; Knierim, 2005; Yoganarasimha and Knierim, 2005). Head direction cells fire selectively when the rat's head is pointed in a particular direction in allocentric space, regardless of its location (Ranck, 1985; Taube et al., 1990a,b; Taube, 1998). Like place cells, head direction cells are controlled by a complex interaction between idiothetic cues and external landmarks (Taube and Burton, 1995; Blair and Sharp, 1996; Goodridge et al., 1998; Wiener et al., 2002; Zugaro et al., 2003). Head direction cells and place cells are usually tightly coupled. A shift in the preferred firing direction of a head direction cell relative to the external environment is accompanied by either an equivalent rotation of CA1 place fields or a remapping of the CA1 representation (Knierim et al., 1995, 1998). Moreover, the preferred firing locations/directions of place cells and head direc- 


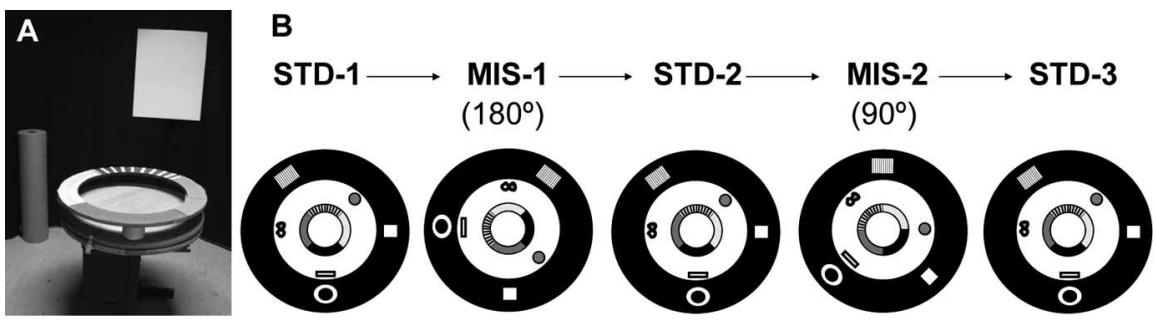

Figure 1. Schematic representation of the circular track location and the cue configuration. $\boldsymbol{A}$, Photograph showing the circular track with salient visual and tactile information (proximal cues) in the center of the room, surrounded by the black curtain with distal landmarks. $\boldsymbol{B}$, The circular track had four different textures as proximal cues (each covering a quadrant of the track) and was placed in the center of the behavioral room, surrounded by a black curtain. The set of distal cues comprised six objects either hanging on the curtain or standing on the floor. Throughout the training, the relationship between proximal and distal cues was that of the standard configuration. During recording sessions, three standard sessions (STD) were interleaved with two mismatch sessions (MIS), in which the cues were rotated in opposite directions (proximal cues CCW; distal cues CW), for a total mismatch of $45^{\circ}, 90^{\circ}, 135^{\circ}$, or $180^{\circ}$. Shown in the figure are examples of $180^{\circ}$ and $90^{\circ}$ mismatch sessions

tion cells remain strongly coupled to each other even when they become completely uncoupled from external landmarks (Knierim et al., 1995).

Most computational models of head direction cells incorporate continuously coupled attractor dynamics, with an angular velocity signal used to shift the "activity bump" on the ring attractor to correspond with the animal's head direction (Skaggs et al., 1995; Blair, 1996; Redish et al., 1996; Zhang, 1996; Song and Wang, 2005). Such an attractor would hinder the split representation of head direction. Because of the coupling between head direction cells and CA1 place cells, it is important to determine whether the head direction cell system maintains internal coherence under conditions in which the CA1 place representation splits, or whether the head direction representation can also split under these circumstances. In the present experiment, ensembles of head direction cells from the anterior thalamus and place cells from CA1 were recorded simultaneously, under the same proximal-distal counter-rotation conditions as published previously for CA1 and CA3 cells (Shapiro et al., 1997; Knierim, 2002a; Lee et al., 2004). The simultaneous recordings from different brain regions allowed direct comparisons between these regions in the same recording sets and showed that the head direction cell ensemble was more coherent than the place cell ensemble under these cue-mismatch conditions.

\section{Materials and Methods \\ Subjects}

Six male Long-Evans rats, aged 5 months and weighing $\sim 600 \mathrm{~g}$ at arrival, were housed individually on a $12 \mathrm{~h}$ reversed light/dark cycle, with access to food and water ad libitum. During behavioral training and recordings, rats were maintained at $80-90 \%$ of their free-feeding weights, and recordings were performed during the dark portion of the light/dark cycle. Animal care, surgical procedures, and killing of the animals were performed in accordance with National Institutes of Health and University of Texas Health Science Center at Houston Institutional Animal Care and Use Committee guidelines.

\section{Surgery}

Under surgical anesthesia, a custom-built recording drive (hyperdrive) allowing the independent manipulation of 20 recording probes was implanted over the right hemisphere. An anterior bundle contained 11 tetrodes aimed at the anterior dorsal nucleus (ADN) of the thalamus, and a posterior bundle contained seven tetrodes aimed at the CA1 layer of the hippocampus. Each bundle also contained a single reference electrode for differential recording. During surgery, electrode recordings were performed to identify the location of the septal pole of the hippocampus, and the front of the anterior bundle was positioned $0.3 \mathrm{~mm}$ anterior to that point and $1.3-1.6 \mathrm{~mm}$ from the midline. This procedure was a more reliable method to center the electrode array over the ADN than relying on position relative to bregma. The posterior bundle was always located $1.6 \mathrm{~mm}$ posterior and $0.3 \mathrm{~mm}$ lateral to the center of the anterior bundle.

\section{Training}

After $7 \mathrm{~d}$ of postsurgical recovery, the rats were trained to run clockwise (CW) on a circular track $(56 \mathrm{~cm}$ inner diameter, $76 \mathrm{~cm}$ outer diameter) for food reward (chocolate sprinkles). The circular track had four different textured surfaces as proximal visual and tactile cues, each covering one quarter of the track: a gray rubber mat with a pebbled surface; brown, mediumgrit sand paper; beige carpet pad material; and gray duct tape with white tape stripes (Fig. 1). The circular track was centered inside a black curtain (275 cm diameter) reaching from ceiling to floor, with some distal cues hanging on the curtain and others standing on the floor at the perimeter of the curtain. The distal cues consisted of six objects: a brown cardboard circle, a white box, an intravenous stand with a lab coat and a blue cloth, a black and white striped card, a roll of brown wrapping paper, and a white card. The ceiling was covered with a black curtain, and the ceiling panel was also painted black. A single, $25 \mathrm{~W}$ bulb on the ceiling was centered over the track, and an intercom speaker was mounted $14 \mathrm{~cm}$ offset from the light for communication between experimenters in the behavior room and in the adjacent computer room, which housed the recording electronic equipment. A white-noise generator was placed directly beneath the small table on which the track stood to mask external sounds. During training sessions, the rat was carried directly into the room on a pedestal, the head stage was connected to the recording cable, and the animal was placed on the track at a random starting point. The experimenter moved around the track frequently so as not to become a stable landmark for the rat, placing chocolate sprinkles at arbitrary locations on the track (approximately two rewards per lap) such that no areas of the track were preferentially associated with the reward (Knierim, 2002a). Attempts by the rat to move counterclockwise (CCW) were discouraged by blocking its progress with a piece of cardboard. Throughout the training sessions, the circular track and the array of distal cues were kept at a constant configuration, as shown in standard (Std) sessions (Fig. 1). Each rat had $30 \mathrm{~min}$ of training daily over a period of $8-12 \mathrm{~d}$, before the start of actual recording sessions.

\section{Recording electronics}

After postsurgical recovery, the tetrodes were slowly advanced over the course of several days as the rat sat quietly in a towel-lined dish on a turntable next to the recording electronics outside the behavioral room. The tetrodes of the posterior bundle were lowered to the CA1 pyramidal cell layer by monitoring the depth profile of hippocampal sharp waves (Buzsaki, 1986) and observing the increase of multiunit activity. The tetrodes of the anterior bundle were lowered to the thalamus, whereupon head direction cell activity was monitored by rotating the rat passively on a turntable after each tetrode adjustment and listening for the characteristic directional tuning of these cells.

Recordings were performed with the Cheetah Data Acquisition System (Neuralynx, Tucson, AZ). Neural signals were amplified (2000-5000 times), filtered ( $600 \mathrm{~Hz}$ to $6 \mathrm{kHz}$ ), digitized at $32 \mathrm{kHz}$, and stored on a personal computer. The head stage had a circular array of light-emitting diodes (LEDs) (five red LEDs in front and five blue LEDS in back) and a boom arm that extended two green LEDs $15 \mathrm{~cm}$ behind the head stage. The output of a color CCD camera (model 1300; Cohu, San Diego, CA) mounted on the ceiling was captured by a video frame grabber (DT3120; Data Translation, Marlboro, MA) at $30 \mathrm{~Hz}$. At each frame, the position of the rat was defined as the center of mass of all blue and red pixels (the LEDs over the rat's head), and the head direction was defined as the angle between the center of mass of the blue and red pixels and the center of mass of the green pixels (the LEDs on the boom arm). 


\section{Experimental protocol}

Baseline data were collected from the rat during sleep or awake immobility for 20-30 min before and after the behavioral sessions on all days to assess the overall recording stability. After the first baseline session, the rat was placed in a covered box and, after $30 \mathrm{~s}$, was taken in the box on a brief walk in the computer room and around the track to disrupt the rat's ability to maintain a strong sense of direction between the behavioral area and the external environment (Knierim et al., 1995; Jeffery and O'Keefe, 1999). The rat was then placed on a pedestal located in the center of the track, the head stage was connected to the recording cables, and the rat was placed on the track at a random starting point while the pedestal was removed. After 15 laps on the track, the rat was placed back on the pedestal, the cables were disconnected, and the rat was placed back in the covered box and again taken on a brief random walk. Each day of recording consisted of five track sessions, with three standard sessions interleaved with two mismatch sessions. Standard sessions maintained the proximal and distal cue configuration as it was during training. In the mismatch sessions, the circular track (proximal cues) was rotated CCW $22.5^{\circ}, 45^{\circ}, 67.5^{\circ}$, or $90^{\circ}$, and all of the distal cues along the curtain were rotated CW by an equal amount, resulting in a total mismatch amount of $45^{\circ}, 90^{\circ}, 135^{\circ}$, or $180^{\circ}$ between proximal and distal cues. Each rat received four sets of each rotation mismatch over $8 \mathrm{~d}$, with each mismatch being run in pseudorandom manner (except for rat 114, which had only one set of each rotation mismatch over $2 \mathrm{~d}$ of recording).

\section{Data analysis}

Off-line unit isolation and selection criteria. All of the data acquired during baseline and recording sessions were analyzed off-line. Single units were isolated based on the relative amplitudes of signals recorded simultaneously at four slightly different locations on the tetrode. Additional waveform characteristics, such as spike width, were also used for isolation. Waveform characteristics were plotted as a scatter plot of one of the channels versus another. Individual units formed clusters of points on such scatter plots, and the boundaries of these plots were defined with the use of a custom interactive program running on a personal computer workstation. Isolation quality of the cell was rated on a subjective scale of 1 (very well isolated) to 4 (marginally isolated), based on the size of the waveforms relative to background and on the closeness and degree of potential overlap between neighboring clusters. These ratings were made completely independent of the place field qualities or preferred head direction of the cell or of its response to the cue manipulations. All of those cells rated "marginally isolated" were excluded from analysis.

Creation of spatial and directional tuning curves. Head direction cell tuning curves were generated by dividing the number of spikes fired when the rat faced a particular direction (in bins of $5^{\circ}$ ) by the total amount of time the rat spent facing that direction on the circular track. For CA1 place cells, the circular track was linearized (in bins of $5^{\circ}$ ) to generate one-dimensional firing-rate arrays by dividing the track into equal-sized position bins and, for each bin, dividing the number of spikes fired by the amount of time the rat occupied that bin. Spikes that occurred when the rat's head was positioned off the track were excluded, because these off-track positions were not sampled consistently across sessions. The quality of spatial tuning for place cells was quantified with the spatial information measure developed by Skaggs et al. $(1993,1996)$. This number, derived from the principles of information theory, reflects the amount of information about the rat's position that is conveyed by the firing of a single spike from the neuron. Under the conditions of the present experiment, a spatial information score of $\geq 1$ bit/spike corresponds well with an experienced observer's judgment of a well tuned, CA1 place cell.

Rotational correlation analysis. To compare how the directional tuning curves of head direction cells and place fields of individual CA1 cells rotated between sessions, the Pearson product-moment correlation between the firing-rate arrays of a cell in the two sessions was measured, and then the firing rate bins of the second session were shifted by one bin (equal to a $5^{\circ}$ shift). The firing-rate array of the first session was correlated with the shifted array of the second session, and then the second array was again shifted by $5^{\circ}$. This was repeated 71 times, and the shift angle that produced the highest correlation was taken as the amount that the place field or head direction tuning curve had rotated between the two sessions. Correlations were computed on all head direction cells that met the isolation criteria and on the CAl cells that met the isolation criteria and met the following place field criteria in both of the two sessions: (1) the spatial information score was $\geq 1.0$ bit; (2) the cell fired $\geq 50$ spikes, a minimum number to ensure reliability of the spatial information score; and (3) the statistical significance of the information score was $p<0.01$ (Skaggs et al., 1993, 1996). Circular statistics were used to calculate the angle and length of the mean vector of the population (Zar, 1999). The angle of the vector represents the mean angle of rotation of the population, and the length of the vector is inversely proportional to the variance of the distribution around that mean.

Probability of apparent cue control by chance. To assess whether the head direction cells were controlled by the proximal cues, the probability $P(X)$ of the observed number of CCW-rotating head direction cell ensembles falling by chance within the range of proximal cue rotation $\left( \pm 22.5^{\circ}\right)$ was analyzed with the following formula (Zar, 1999):

$$
P(X)=\frac{n !}{X !(n-X) !} p^{X} q^{n-X},
$$

where $p$ is the probability that the ensemble would fall by chance within the $\pm 22.5^{\circ}$ range of proximal cue rotation (i.e., $45^{\circ} / 180^{\circ}=0.25$ ), $q$ is the probability that the ensemble would fall by chance outside the $\pm 22.5^{\circ}$ range of proximal cue rotation (i.e., 0.75 ), $n$ is the total number of samples (CCW-rotating head direction ensembles), and $X$ is the number of samples that fell within the $\pm 22.5^{\circ}$ range of proximal cue rotation.

\section{Histology}

On completion of the experiments, marker lesions were made on a subset of the tetrode tips by passing $10 \mu \mathrm{A}$ current for $10 \mathrm{~s}, 1 \mathrm{~d}$ before the perfusion. The rats were perfused transcardially with $4 \%$ Formalin, after which the brain was extracted and kept in $30 \%$ sucrose Formalin solution until the brain sank in the solution. Coronal sections of $40 \mu \mathrm{m}$ thickness were cut on a microtome, mounted, and stained with $0.1 \%$ cresyl violet. Digital photomicrographs were taken for all serial sections, and the distance from midline to the tetrode track markings in each brain section was calculated and plotted. The resulting configuration of electrode tracks was then compared with the configuration of the tetrode bundles and marker lesions to identify which track corresponded to which tetrode. Finally, a depth reconstruction of the tetrode track was performed for each recording session to identify the brain region in which the cells were recorded at that depth.

\section{Results}

Multiple single units from the hippocampus and the anterior thalamus were simultaneously recorded in six rats. On average, three head direction cells (range of two to seven) and 11 CA1 cells (range of 2-33) that met the place field criteria in at least one of the five sessions were recorded each day. Because of the strong possibility that a large fraction of both head direction cells and place cells were recorded multiple times over days, the total number of cells recorded over time is difficult to establish with certainty, and no attempt was made to do so. Most place cell data points (902 of 940,96\%) were localized to the CA1 layer of the hippocampus (Fig. $2 \mathrm{~A}$ ), whereas the remainder (including all of the cells from rat 86 ) were from the CA3 region or the dentate gyrus. All quantitative analyses were restricted to the CA1 cells, because there were not enough cells from CA3 or the dentate gyrus to make meaningful comparisons. Most of the head direction cell data points ( 103 of $133,77 \%$ ) were localized to the ADN of the thalamus (Fig. $2 B$ ). A few units from rat 95 were at the border between the ADN and the lateral dorsal nucleus (LDN) and could not be localized decisively to either region (5 of 133, $4 \%$ ). A minority ( 25 of $133,19 \%$ ) of the data points were localized to the anterior ventral nucleus (AVN), the LDN, and to regions near the border of the AVN and the ventral anterior 

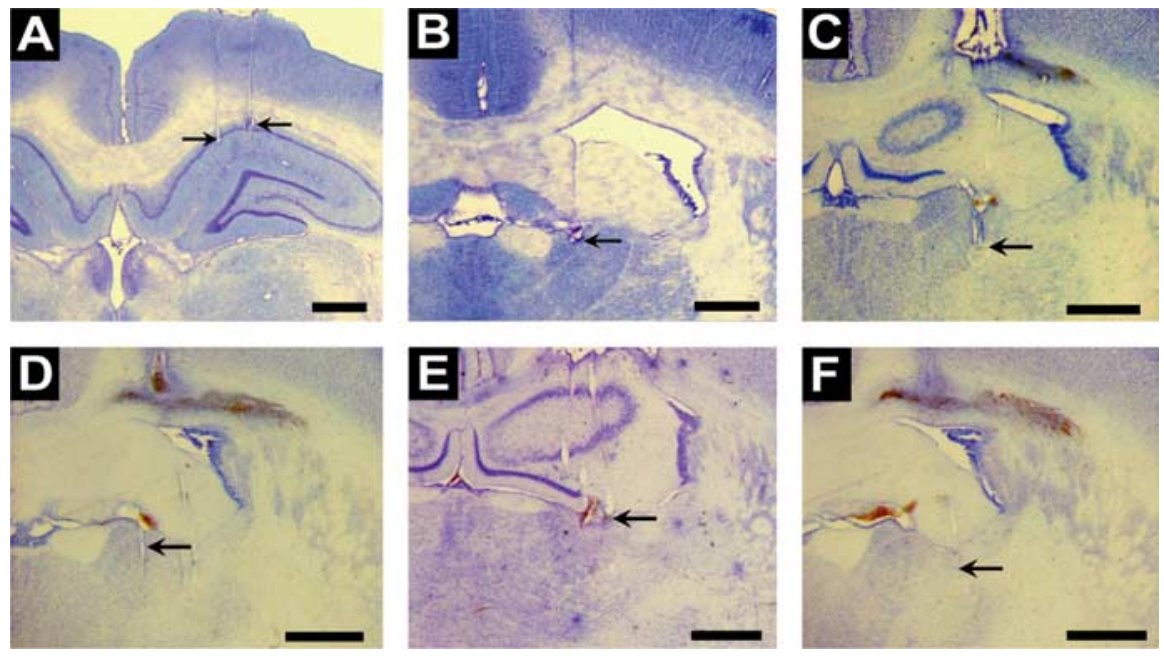

Figure 2. Cresyl violet-stained coronal sections showing representative electrode tracks and recording sites (arrows). $A$, Hippocampal $C A 1$ region. $\boldsymbol{B}, A D N$ of the thalamus. The majority of head direction cells were recorded from ADN. $\boldsymbol{C}-\boldsymbol{E}$, In some rats, head direction units were recorded from the border region between the AVN and VAN of the thalamus $(\boldsymbol{C})$, the AVN of the thalamus $(\boldsymbol{D})$, the LDN of the thalamus $(\boldsymbol{E})$, and the border region between the AVN and RT $(\boldsymbol{F})$. Scale bars, $1 \mathrm{~mm}$.

nucleus (VAN) and near the border of the AVN and the reticular thalamic (RT) nucleus of the thalamus (Fig. 2C-F). All of the recording sites clearly outside the $\mathrm{ADN}$ came from rats 64 and 86. There were no differences between the head direction cells recorded from $\mathrm{ADN}$ and those of other regions, in terms of rotational amounts following cue rotations (see below) and tuning curve parameters in the Std 1 session, such as the maximum rate $(\mathrm{ADN}, 71.19 \pm 25.72$; non-ADN, $80.20 \pm 37.94$; mean $\pm \mathrm{SD}$ ) and the width of the tuning curve at half-height (ADN, $49.90 \pm$ 12.55; non-ADN, $50.80 \pm 14.33$; mean $\pm \mathrm{SD}$ ). Waveform characteristics, such as spike height (microvolts peak to valley) (ADN, $131.52 \pm 42.39$; non-ADN, $128.44 \pm 41.92)$ and spike width (microseconds peak to valley) (ADN, $260.11 \pm 75.48$; non-ADN, $269.23 \pm 104.84)$ also did not vary significantly, although the non-ADN units tended to have an initial positive deflection in their waveforms that preceded the larger negative peak of the potential (see Discussion). This waveform shape was also present in a small minority of ADN units.

Data were obtained over 45 recording days from six rats $(8-10$ $\mathrm{d}$ per rat, except for rat 114 , which had only $2 \mathrm{~d}$ recording, and rat 113 , for which the second day of data was lost because of technical problems). Five recording sessions were run each day, with three standard sessions interleaved with two mismatch sessions, resulting in a total of 135 standard and 90 mismatch sessions. Based on the place field criteria described in Materials and Methods, of all the place cells recorded from CA1, 45\% (403 of 902) maintained a qualified place field in both the standard and mismatch sessions, $13 \%$ (121 of 902) had a qualified place field in the mismatch session only, 30\% (272 of 902) had a qualified place field in the standard session only, and 12\% (106 of 902 data points) had no qualified field in both standard or mismatch session being compared (but they had a qualified place field in at least one of the other sessions recorded that day). This amount of remapping was similar to previous experiments using this manipulation (Knierim, 2002a; Lee et al., 2004). Only those place cells that maintained a qualified place field in both the standard session and the immediately following mismatch session were subjected to additional analysis.

Figure 3 shows an example of simultaneously recorded CA1 place cells and ADN head direction cells in standard and misment in five simultaneously recorded ADN head direction cells, because all of the head direction tuning curves rotated CW in the mismatch sessions, following the rotations of the distal cues. Along with the head direction cells, 19 CA1 place cells were recorded simultaneously in these sessions, of which 10 cells maintained qualified place fields in both standard and mismatch sessions and the remainder remapped. In the first mismatch session, the place cells showed a split representation, because five place fields rotated CW, five place fields rotated CCW, and nine place fields remapped. In the second mismatch session, nine place fields rotated CW, one place field rotated CCW, and nine place fields remapped.

\section{Rotation correlation analysis}

The amount of rotation of the place fields and head direction tuning curves was quantified for the subset of CA1 place cells that maintained place fields in both standard and mismatch sessions (403 of 902 data points) and for all fairly isolated head direction cells ( 262 data points). Figure 5 represents the amount of rotation of all of the place fields and head direction tuning curves between standard sessions and between standard and mismatch sessions. For place fields between the standard sessions, the angle of the mean vector did not deviate significantly from zero (Std $1-3$, mean angle of $-1.6^{\circ}, 95 \%$ confidence interval of $-3.8^{\circ}$ to $0.5^{\circ}$; Std 3-5, mean angle of $-1.1^{\circ}, 95 \%$ confidence interval of $-2.7^{\circ}$ to $0.6^{\circ}$ ), and the length of the mean vector signified that the angles were significantly clustered (Rayleigh's test; Std 1-3, $r=0.95, p<$ 0.0001 ; Std 3-5, $r=0.97, p<0.0001$ ). For head direction cells between the standard sessions, the angle of the mean vector was also close to or equal to zero (Std 1-3, mean angle of $-5^{\circ}, 95 \%$ confidence interval of $-9.2^{\circ}$ to $-1.5^{\circ}$; Std $3-5$, mean angle of $0^{\circ}$, $95 \%$ confidence interval of $-1.0^{\circ}$ to $0.8^{\circ}$ ), and the length of the mean vector signified that the angles were significantly clustered (Rayleigh's test; Std 1-3, $r=0.93, p<0.0001$; Std 3-5, $r=0.99$, $p<0.0001)$. These results indicate that the representations of the standard environment were stable between sessions (although in two cases head direction cell ensembles were not stable between Std 1 and Std 3, resulting in the two clusters of outliers for the Std 1-3 head direction cell diagram of Fig. 5). In different mismatch sessions, the mean angle of rotation of the place cell population 
deviated from zero $\left(7^{\circ}, 34^{\circ}, 60^{\circ}\right.$, and $78^{\circ}$ in the $\pm 22.5^{\circ}, \pm 45^{\circ}, \pm 67.5^{\circ}$, and $\pm 90^{\circ}$ mismatch sessions, respectively; in all cases, the $95 \%$ confidence interval excluded $0^{\circ}$ ), showing that the population of place fields rotated increasingly as a result of the increasing cue mismatch (Watson-Williams test, $\left.F_{(3,402)}=21.47 ; p<0.001\right)$. However, the length of the mean vector decreased with increasing mismatch amounts for place fields $(r=0.90,0.45,0.45$, and 0.30 in the $\pm 22.5^{\circ}, \pm 45^{\circ}, \pm 67.5^{\circ}$, and $\pm 90^{\circ}$ mismatch sessions, respectively; Rayleigh's test, $p<0.001$ for each vector), indicating an increasing variance of the distribution around that mean (KruskalWallis test on angular distance from mean, $H=39.96 ; p<0.0001)$. The mean angle of rotation of head direction cells also deviated from zero $\left(19^{\circ}, 42^{\circ}, 50^{\circ}\right.$, and $68^{\circ}$ in the $\pm 22.5^{\circ}, \pm 45^{\circ}, \pm 67.5^{\circ}$, and $\pm 90^{\circ}$ mismatch sessions, respectively; in all cases, the $95 \%$ confidence interval excluded $0^{\circ}$ ), showing that the population of head direction cells rotated increasingly as a result of the increasing cue mismatch (WatsonWilliams test, $\left.F_{(3,252)}=30.21 ; p<0.001\right)$. The vector lengths of the head direction cell population $(r=0.94,0.96,0.93$, and 0.68 in the $\pm 22.5^{\circ}, \pm 45^{\circ}, \pm 67.5^{\circ}$, and $\pm 90^{\circ}$ mismatch sessions, respectively; Rayleigh's test, $p<0.0001$ for each vector) were not the same for each mismatch session (Kruskal-Wallis test on angular distance from mean, $H=50.10 ; p<0.0001$ ), indicating that the scatter of rotation angles around the mean were different for different mismatch sessions.

\section{Split representation}

In the analysis shown in Figure 5, some place cells and head direction cells rotated their tuning curves CW to follow the distal landmarks, and other place cells and head direction cells rotated their tuning curves CCW to follow the proximal cues. It is possible that individual datasets contained a subset of place fields that followed the distal cues, whereas another subset simultaneously followed proximal cues. Alternatively, these distributions might be an artifact of the pooling of individual datasets in which all simultaneously recorded place fields followed either one set of cues or other, and the dominant set of cues changed between sessions. To evaluate whether split representations of the mismatch environments occurred in either the place cells or head direction cells, we addressed this issue in two steps.

First, for the mismatch sessions in which multiple well isolated place cells and/or head direction cells were recorded simultaneously, we quantified how many sessions showed evidence of a split representation of location or head direction (Fig. 6). In 75\% of the mismatch sessions, the CA1 place field representation split, with some fields rotating in one direction (usually CW; see Fig. 5) and at least one place field rotating in the opposite direction (regardless of the magnitude of the rotation). In other sessions, all
STD - $1 \quad 180^{\circ}$-MIS STD - $2 \quad 135^{\circ}$-MIS STD - 3
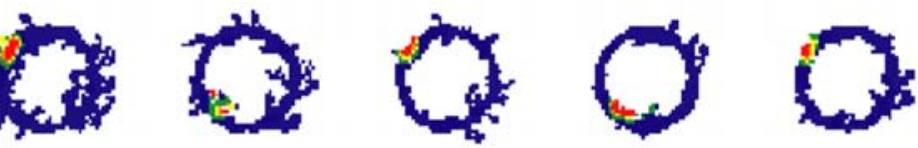

22.45
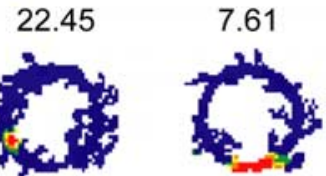

28.38

10.22

31.09
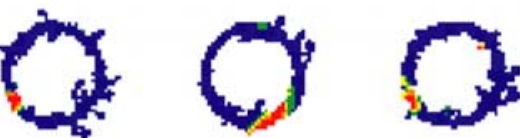

20.72

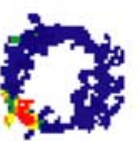

18.40

16.66

14.01

10.00
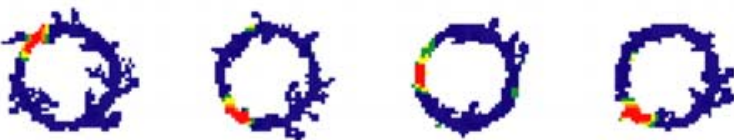

18.09

16.48

21.89

15.95

22.08
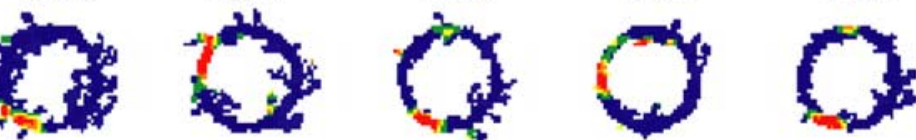

19.47

20.00

15.18

12.58
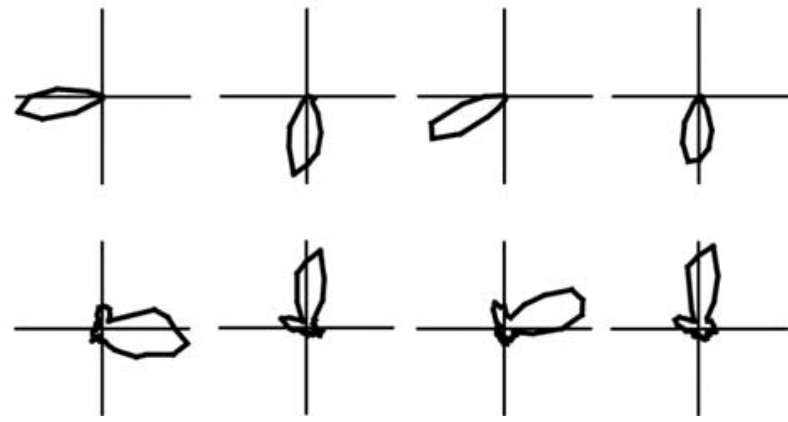

Figure 3. Representative examples of simultaneously recorded CA1 place field rate maps and head direction cell tuning curves across standard and mismatch sessions. In mismatch sessions, some place fields followed the CCW proximal cue rotation (11903recording. (Numbers below each place field rate map represent maximum firing rates in Hertz.) However, unlike place cells, all of 作 windicate rat number (first three digits) and day of recording, followed by tetrode and cell number. For the color code, red indicates $>90 \%$ of the peak firing rate for that cell, blue indicates no firing, and intervening colors of the spectrum indicate successive decrements of $10 \%$ of the peak firing rate.

of the place fields rotated either CW (15\%) or CCW (10\%). In contrast, the head direction cells never showed split representations of the mismatch environment, because they always rotated coherently as an ensemble either CW (94\%) or CCW (6\%). The difference between the place cell representations and the head direction cell representations was highly significant $\left(\chi^{2}=100.66\right.$; $p<0.0001)$. Of the 66 ensembles in which two or more head direction cells were recorded simultaneously between standard and mismatch sessions, all head direction tuning curves rotated within $5^{\circ}$ of each other in $61 \%$ of the cases ( 40 of 66 ) and within $20^{\circ}$ of each other in $95 \%$ of the cases ( 63 of 66 ). The largest spread was a single ensemble in which the range of tuning curve rotations was $30^{\circ}$, which we believe is within the range of measurement error from biases in LED occlusions at different parts of the circular track. Furthermore, of the 225 total recording sessions (standard and mismatch), there was not a single instance in which, between sessions, one or more head direction cell tuning curves rotated in the opposite direction from the other simulta- 


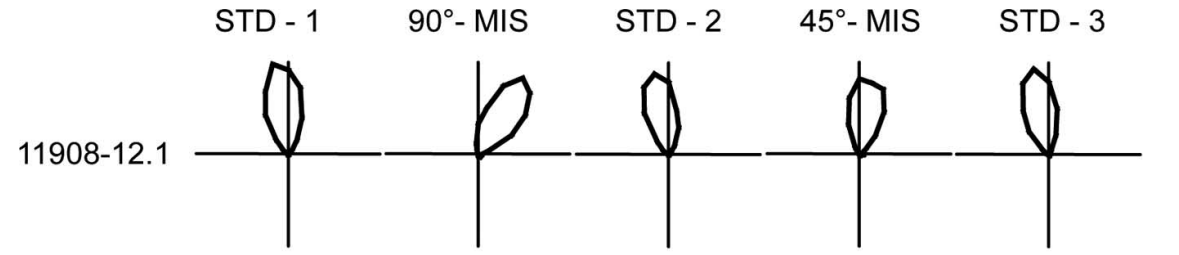

$11908-12.2$

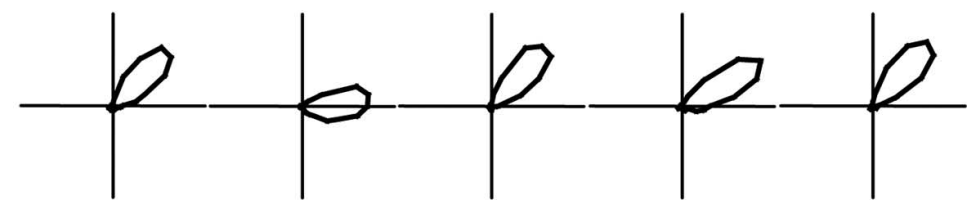

$11908-13.1$

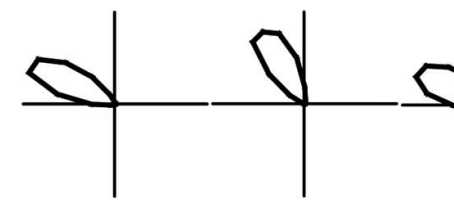

$11908-13.2$
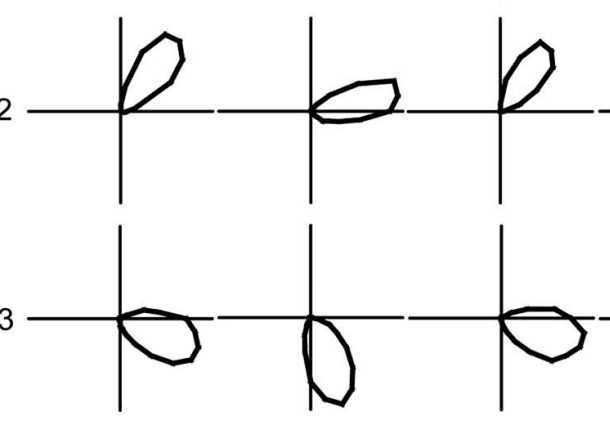

Figure 4. Rotational coherence of head direction cells. Shown are the five head direction cell tuning curves recorded simultaneously in a single recording session across standard (STD) and mismatch (MIS) conditions from the ADN. All five head direction tuning curves shifted their preferred firing direction $\mathrm{CW}$ by an amount equal to the rotation of distal cues. (The axes for each cell are scaled as follows: $11908-12.1,112 \mathrm{~Hz} ; 11908-12.2,41 \mathrm{~Hz} ; 11908-13.1,110 \mathrm{~Hz} ; 11908-13.2,80 \mathrm{~Hz} ; 11908-13.3,156 \mathrm{~Hz}$.) Numbers preceding each row indicate rat number (first three digits) and day of recording, followed by tetrode and cell number.

CA1

A
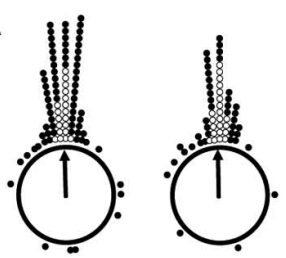

B
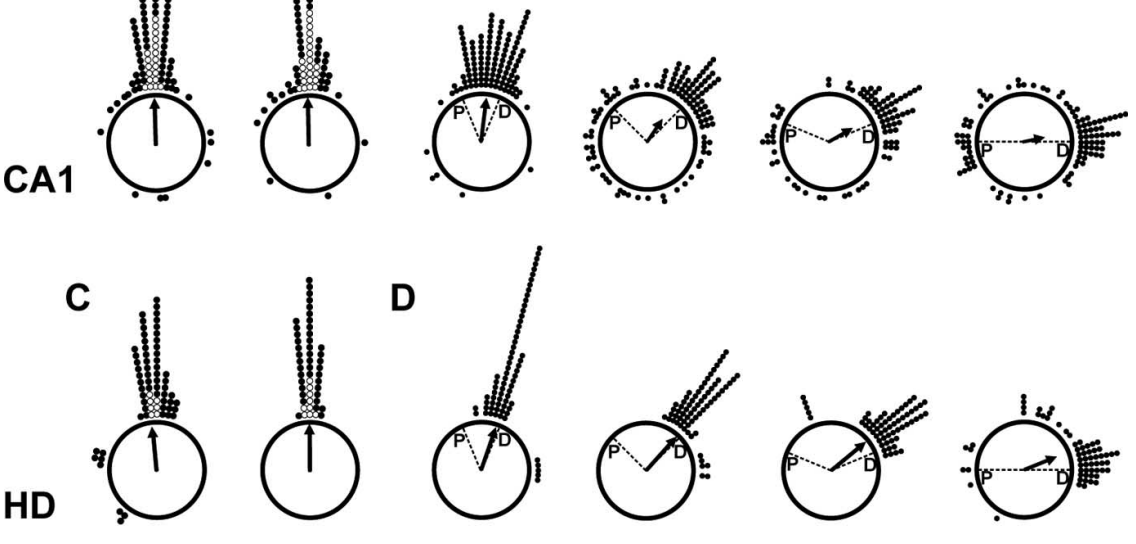

STD 1-3 STD 3-5

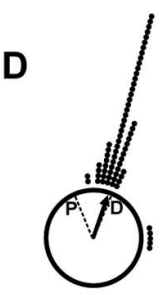

MIS-45

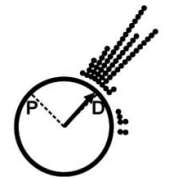

MIS-90

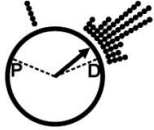

MIS-135

Figure 5. Summary of CA1 place field and head direction cell tuning curve rotations. Each dot on the polar plot (open dots, 10 data points; filled dots, 1 data point) represents the amount of rotation of a CA1 place field and a head direction cell tuning curve between two standard sessions $(\boldsymbol{A}, \boldsymbol{C})$ and between standard (STD) versus differentmismatch (MIS) sessions $\left(45^{\circ}, 90^{\circ}, 135^{\circ}\right.$, and $\left.180^{\circ}\right)(\boldsymbol{B}, \boldsymbol{D})$. The arrows represent the mean angle of rotation of the population, and the length of the arrow represents the angular dispersion around the mean. The dashed lines indicate the rotation amounts of the proximal ( $(\mathrm{CW})$ and distal ( $\mathrm{CW})$ cue sets. Because cells were recorded over multiple sessions and days, each dot does not correspond to a unique cell. P, Proximal cue; D, distal cue.

neously recorded tuning curves. These results indicate that the head direction cells always maintained a strong coherence of their representation, even under conditions in which CA1 place cells created split representations.

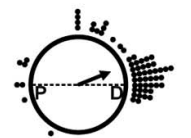

MIS-180
Previous studies using this cue manipulation have reported a large degree of intersubject variability, in that some rats had CA1 place fields that were predominantly controlled by the proximal cues and other rats had CA1 place fields that were predominantly controlled by the distal cues (Knierim, 2002a; Lee et al., 2004). In the present study, most CA1 place fields were controlled by the distal landmarks, and thus the amount of split representation of CA1 cells shown in Figure 5 appears less than that reported in these previous studies. To determine whether, at the level of single datasets, the amount of split control was comparable with the previous studies, we evaluated whether the split representation of place fields occurred to a degree greater than expected by random remapping (Fig. 7). That is, because CA1 demonstrated a partial remapping, in which many cells became silent or developed new fields, it is possible that the place fields that rotated CCW by the same amount as the proximal cues were actually place fields that remapped and, by chance, the remapped fields corresponded to the rotation of the proximal cues. To test this possibility, for each session, the dominant set of cues was determined by comparing the number of place fields that rotated CW or CCW, and then those place fields controlled by the dominant set of cues were dropped from the analysis. (If the number of CW-rotating fields equaled the number of CCW-rotating fields, then that entire session was dropped from the analysis.) The remaining minority of cells (88 cells) formed the group that was not controlled by the dominant set of cues. If these place fields remapped between sessions, then their rotation angles should fall randomly within the $0-180^{\circ}$ range of rotation of the nondominant cue set; in contrast, if these place cells were indeed controlled by the nondominant set of cues, then their place fields should cluster within the $45^{\circ}$ range centered on the rotation of that cue set. According to the null hypothesis that the fields remapped to random locations from 0 to $180^{\circ}$, the expected number of cells in the $45^{\circ}$ cue-controlled range would be $25 \%$ (i.e., $45^{\circ} / 180^{\circ}$ ) of the total number of cells (22 of 88 cells), and the expected number of cells outside of this $45^{\circ}$ range would be $75 \%$ of the total number of cells ( 66 of 88 cells). Instead, $50 \%$ ( 44 of 88 ) of the total number of fields fell within the $45^{\circ}$ range of nondominant cue control, and 50\% (44 of 88) of the fields fell outside of this range, which was different from the expected values by the assumption of uniform distribution $\left(\chi^{2}=28.35 ; p<0.0001\right)$. These numbers are nearly identical to those produced by the same analysis in a previous paper (Knierim, 


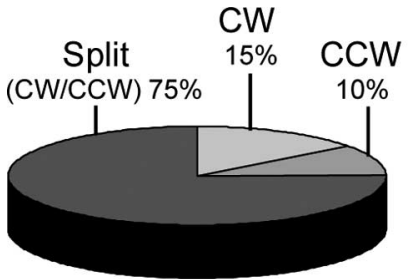

CA1

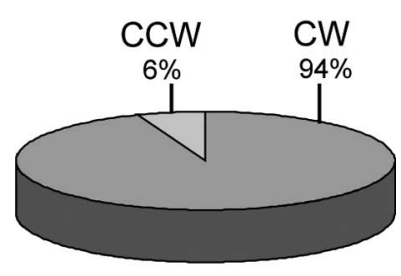

HD
Figure 6. Split representation in mismatch sessions. A total of 90 mismatch sessions were recorded out of 225 recording sessions. In $75 \%$ of the mismatch sessions, the hippocampal CA1 ensembles revealed split representations in which some place fields rotated $\mathrm{CW}$ and some rotated CCW in the same dataset. In contrast, the head direction (HD) cells always rotated either CW $(94 \%)$ or CCW (6\%) as an ensemble in mismatch sessions, never showing a split representation of head direction.

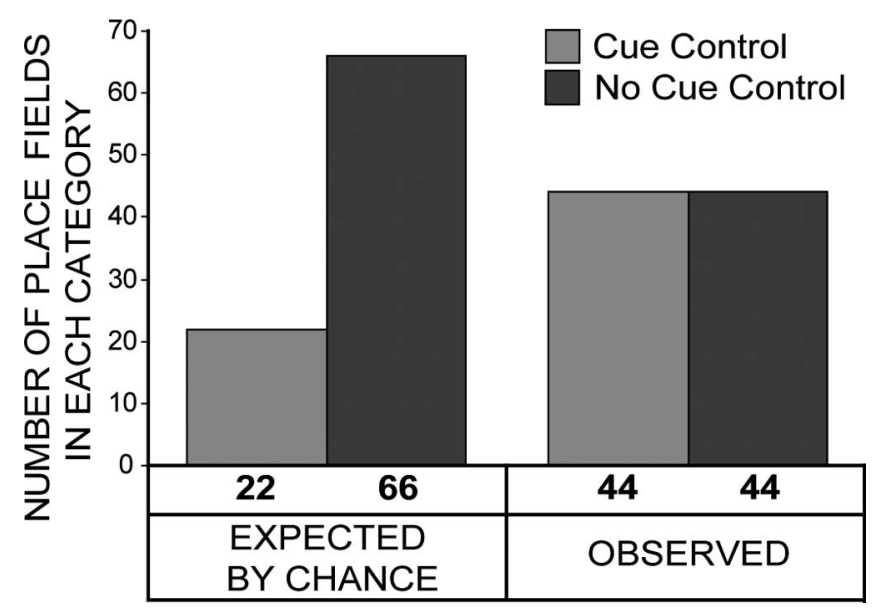

Figure 7. Split control of CA1 place cell ensembles. The occurrence of partial remapping in place cells might contribute to the observed split representation by place cells if randomly remapped fields happen to fall within the range of nondominant cue rotation. To rule out this possibility, the data were statistically analyzed on 88 cells that formed a group that was not controlled by the dominant set of cues. If these cells were controlled by the nondominant set of cues, then their place fields should cluster within the $45^{\circ}$ range centered on the rotation of that cue set. Alternatively, if they remap to arbitrary angles, then the location of their place fields should be distributed randomly. One-half of the place fields rotated within the $45^{\circ}$ range of nondominant cue control, and the other half rotated outside this range, which is different from the expected chance distribution $\left(\chi^{2}=28.35 ; p<0.0001\right)$.

2002a), demonstrating that the split representation of the place cells indeed occurred at the level of individual datasets and legitimizing the contrast with the coherent representation of the head direction cells.

\section{Proximal cue control over head direction cells}

Distal landmarks apparently have stronger control over head direction cells than do proximal landmarks (Zugaro et al., 2001). This preference was true in the current study (Figs. 5, 6). One question that arises is whether the head direction cells remained coherent because, unlike the CA1 place cell system, perhaps the head direction cells do not receive input from neural representations of proximal landmarks. Although Figure 5 shows that some head direction cell ensembles apparently rotated with the proximal cues (especially in the $180^{\circ}$ mismatch session), it is possible that these sessions were ones in which the head direction ensemble bearing rotated randomly away from the distal landmarks and rotated by chance to the direction defined by the proximal cue rotation. To address this question, we analyzed the five ensembles in which the head direction cells rotated their tuning curves CCW (in the direction of the proximal cue rotation). In three of these five cases, the mean rotation angle fell within the $45^{\circ}$ range centered on the actual rotation angle of the proximal cues. The probability of this occurring by chance is 0.09 (Zar, 1999). Furthermore, in all three cases, the simultaneously recorded place cells were also within the $45^{\circ}$ range of proximal cue control. The probability of this occurring by chance is only 0.02 . Thus, in these datasets, it is unlikely that the place cells and head direction cell tuning curves showed proximal cue control strictly by chance. Rather, the proximal cues exerted a relatively weak, but significant, effect on the head direction ensemble, consistent with a previous report that the head direction cell orientation can be controlled by the orientation of a T-maze (Dudchenko et al., 2005) (see also Zugaro et al., 2004).

\section{Coherence of head direction tuning curves across different thalamic nuclei}

Although the small numbers of cells recorded outside the ADN from two rats does not warrant a statistical analysis, it is noteworthy that we saw no strong evidence that the cells outside the ADN behaved any differently from the cells within the ADN. In the four mismatch sessions in which an ADN head direction cell was recorded simultaneously with an $\mathrm{LDN}$ head direction cell, the tuning curves rotated coherently within $5^{\circ}$ of each other. In general, the units recorded outside the ADN tended to be controlled as a coherent ensemble by the distal cues just like the units in the ADN. Moreover, there was no obvious difference between the $\mathrm{ADN}$ units and the non-ADN units in terms of the probability of the tuning curves becoming decoupled (i.e., $>22.5^{\circ}$ deviation) from either set of controlled cues (i.e., the outliers in Fig. 5). In the eight cases of such loss of cue control, two ensembles contained non-ADN units, and all simultaneously recorded tuning curves rotated within $20^{\circ}$ of each other.

\section{Discussion}

\section{Proximal versus distal landmarks}

The present study demonstrates that, when salient proximal and distal cues are rotated in opposite directions, the CA1 place cell representation splits and remaps, whereas the head direction representation in the thalamus maintains an internally coherent representation that is strongly bound to the distal landmarks. Consistent with previous studies (Shapiro et al., 1997; Tanila et al., 1997; Fenton et al., 2000; Zinyuk et al., 2000; Knierim, 2002a; Lee et al., 2004), some place fields followed the distal cues, some followed the proximal cues, some fields split into two, and other fields remapped. In contrast, all of the head direction cells acted as a coherent ensemble, because they followed the distal cue rotations in most of the mismatch sessions. In the few remaining sessions, either they followed the proximal cue rotation or they rotated to an angle outside the range of actual cue rotation. However, unlike place fields, split head direction representations of the mismatch environment were never observed (Taube and Burton, 1995; Zugaro et al., 2001).

Previous reports have suggested that place cells and head direction cells are closely coupled, in that rotations of the salient landmarks cause relatively equal rotations of both the place fields and the head direction cell tuning curves (O'Keefe and Conway, 1978; Muller and Kubie, 1987; Taube et al., 1990b; Knierim et al., 1998; Knierim, 2005). The cells remain strongly coupled to each other, even when they become completely uncoupled from external sensory cues (Knierim et al., 1995). Although previous studies have shown that individual place fields can become uncoupled 


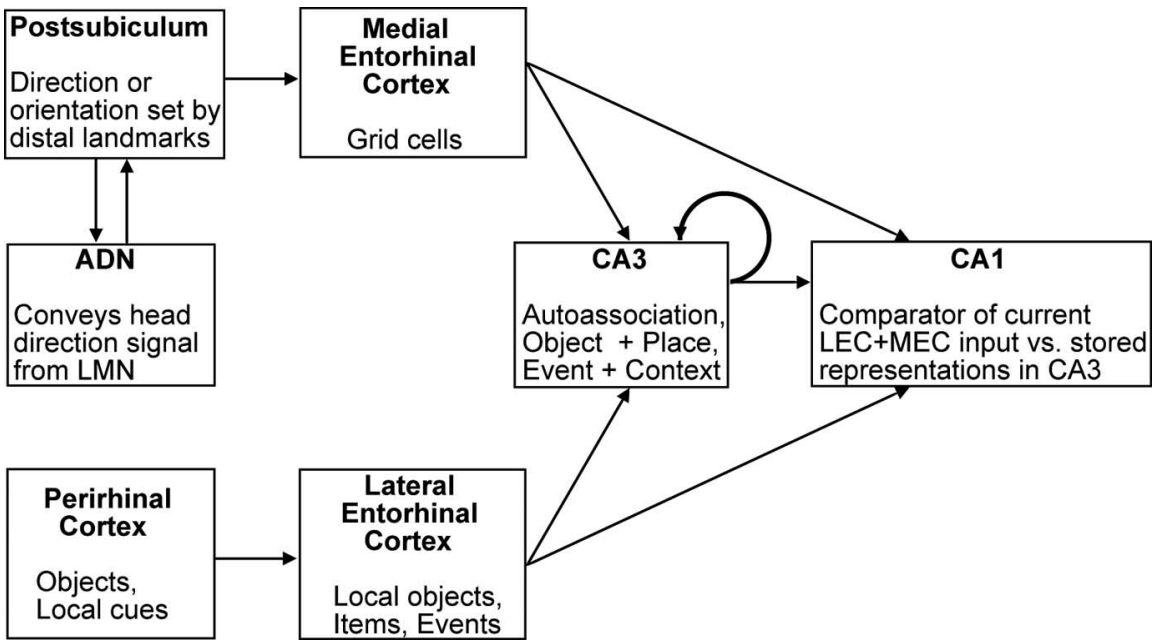

Figure 8. Hypothetical pathways by which proximal and distal cue information may reach the hippocampus. Distal landmarks may provide orientation information via head direction cell control over medial entorhinal input into the hippocampus. Proximal cues are hypothesized to exert control via an object/item pathway through the lateral entorhinal cortex.

represents a consistent preference of CA3 for proximal cues or merely reflects the stochastic nature of individual differences between rats (i.e., CA3 of another group of rats might have shown a distal-cue preference, as the result of inherent differences, small differences in training history, etc.).

\section{Head direction units in multiple thalamic nuclei}

The majority of head direction cells recorded in the present study was from the $\mathrm{ADN}$ of the thalamus. However, we also observed head direction units in other thalamic nuclei. There was no significant difference between the ADN and non-ADN head direction units in such waveform characteristics as spike height and spike width, although the non-ADN units were more likely than the ADN units to show an initial positive deflection in the waveform.

(at least transiently) from the head direction cell system if the place fields remap (Knierim et al., 1995, 1998), the present study shows that a significant minority of place cells can maintain their place fields, but the fields can rotate in the direction opposite to the rotation of the head direction cell representation and the majority of the other place fields. Thus, the premise that the orientation of the hippocampus place representation is determined by the head direction cell system must be modified to some degree (McNaughton et al., 1996; Muller et al., 1996; O’Keefe and Burgess, 1996; Knierim, 2005; Yoganarasimha and Knierim, 2005). Nonetheless, no experiment has yet reported that a coherent CA1 place cell representation can become uncoupled from the head direction representation.

Lee et al. (2004) showed that the CA3 place field representation was more coherent than CA1 when the same mismatch manipulation was done as in the present experiment. The CA3 representation was not completely coherent, however, because a significant minority of place fields remapped or rotated with the nondominant cue set. Most models of the head direction system contain an attractor neural network at the core of the system (Skaggs et al., 1995; Blair, 1996; Redish et al., 1996; Zhang, 1996; Song and Wang, 2005). The complete coherence of the head direction system in the present experiment is consistent with these models. Although a hypothetical split of the head direction representation would not necessarily disprove the attractor models [because of the hypothesized learned external inputs from spatial landmarks (Skaggs et al., 1995; Knierim, 2002a)] (see also Stringer et al., 2004), these results suggest that the internal attractor dynamics of the head direction cell system may be stronger than that of either CA3 or CA1. An alternative possibility is that the head direction cells receive only weak input from the representations of proximal cues, thereby making these cues unable to exert a strong "pull" on the system and cause the representation to split.

The CA3 place cells of Lee et al. (2004) tended to be controlled by the proximal cues, whereas the head direction cells of the present study tended to be controlled by the distal cues. Although a growing body of evidence suggests that the head direction cells and the orientation of the place cell representation are more strongly controlled by distal than proximal cues (Cressant et al., 1997; Zugaro et al., 2001), it is not known whether the proximalcue preference of the CA3 cells in the study by Lee et al. (2004)
The waveform shape of the extracellularly recorded action potential can depend on the proximity of the recording tetrode to different parts of the neuronal soma (Humphrey and Schmidt, 1990), but it can also depend on whether the recording was from the soma or from a fiber of passage. Waveform parameters can also systematically vary between excitatory projection neurons and inhibitory interneurons. It is therefore not certain whether the non-ADN units were excitatory somas, inhibitory somas, or fibers of passage. Future work that targets these regions specifically will be needed to determine whether these thalamic nuclei (other than the ADN and LDN) (Mizumori and Williams, 1993; Taube 1995) contain head direction cells, or whether they merely contain axons that pass through or terminate in these regions.

Previous reports were ambiguous as to whether the AVN contained head direction cells (Taube, 1995; Calton et al., 2003), but one tetrode track in the present study clearly localized a head direction unit to the AVN (Fig. 2D). We also observed head direction units at the border regions between AVN and VAN. The VAN has major connections with rostral premotor areas and the basal ganglia (McFarland and Haber, 2002), and head direction cells have been recorded in the striatum (Wiener, 1993; Ragozzino et al., 2001). These pathways might play a role in the control of head direction cells by motor efference copy (Stackman et al., 2003). The head direction units recorded from these thalamic nuclei appear to work as a coherent ensemble. It is not known whether cortical head direction cells in the postsubiculum or retrosplenial cortex are also part of this coherent representation. The postsubiculum appears to be the location in which visual landmarks become associated with head direction cells during exploration of a novel environment, and postsubiculum head direction cells may convey this association to the ADN via feedback projections (Goodridge and Taube, 1997). Because the postsubiculum also presumably feeds the head direction signal to the hippocampus via the entorhinal cortex, it is important to determine whether postsubiculum head direction cells remain coherent with the thalamic head direction cells or whether the postsubiculum representation may split in the same manner that the CA1 place cell representation splits. The latter result might indicate that the orientations of both proximal-cue-dominated and distal-cue-dominated place cells are controlled by postsubiculum head direction cells. 


\section{Parallel input streams into hippocampus}

Figure 8 presents a speculative model of the pathways by which the proximal and distal cues may influence the CA1 and CA3 place cell representations. Head direction cells in the ADN derive their properties from the lateral mammillary nucleus, which appears to be the origin of the head direction signal together with the dorsal tegmental nucleus (Taube, 1998; Sharp et al., 2001). The postsubiculum associates the head direction signal with the distal landmarks to keep the signal oriented with the external environment, sends feedback to the ADN, and also projects the head direction signal forward to the medial entorhinal cortex (MEC) (Knierim et al., 1995; Goodridge and Taube, 1997; Goodridge et al., 1998; Zugaro et al., 2001). This feedforward signal sets the orientation of the "grid cells" of the MEC (Hafting et al., 2005), which may be the purely spatial input that underlies the place cell firing properties of the hippocampus proper (O'Keefe and Burgess, 2005). The orientation of spatially tuned neurons of the MEC has been shown to correlate with the orientation of ADN head direction cells and with simultaneously recorded CA1 place cells (Yoganarasimha et al., 2003). Thus, the distal landmarks may gain control over the CA1 and CA3 place cells via a multisynaptic pathway starting with the head direction cell circuit, in agreement with arguments that distal landmarks are more appropriate for defining the overall orientation of a spatial map rather than defining precise locations on the map (O'Keefe and Nadel, 1978; Yoganarasimha and Knierim, 2005).

The lateral entorhinal cortex (LEC) apparently does not carry a strong spatial signal when the rat is performing a random foraging task in an environment with a single cue card as a polarizing cue (Hargreaves et al., 2005). The LEC receives major input from the perirhinal cortex, which has been implicated in representations of single items or configurations of stimuli (Brown and Aggleton, 2001; Lindquist et al., 2004). We hypothesize that LEC neurons may be the pathway by which the proximal cue information reaches the hippocampus, because the salient cues on the track (which the rats can touch, smell, and investigate with their vibrissae) may be represented by the LEC neurons as if they were objects. (This prediction will be tested in future experiments.) Thus, CA3 and CA1 may receive information about the orientation of the distal landmarks from MEC and information about the orientation of local cues from the LEC. In the cue-mismatch sessions, CA3 receives the two conflicting types of input and generates an output representation that reduces the conflict by favoring one of the inputs over the other based on the pattern of synaptic weights in its recurrent collateral system that was stored during previous experience in the standard environment (Lee et al., 2004). CA1 also receives the conflicting information from the LEC and the MEC, as well as the more coherent representation from CA3. Under circumstances that are not well understood, sometimes CA1 will be driven by the CA3 input, but on other occasions will be driven primarily by the direct entorhinal input (Brun et al., 2002; Guzowski et al., 2004). Because CA1 lacks a recurrent collateral system, the CA1 place cells result in a split representation when the entorhinal input constitutes the primary drive. This working model, albeit rudimentary, is consistent with a number of studies from rats, monkeys, and humans suggesting a dissociation between object-related (or item-related) information entering the hippocampus via the perirhinal cortex $\rightarrow$ lateral entorhinal cortex pathway and spatial/contextual/source information reaching the hippocampus via the postrhinal (parahippocampal) cortex $\rightarrow$ medial entorhinal cortex pathway (Davachi et al., 2003; Alvarado and Bachevalier, 2005; Norman and Eacott, 2005). These representations might then be transformed into conjunctive object-place (or item-context) representations in the hippocampus, which may set the foundation for representations necessary for context-dependent learning and episodic memory (O'Keefe and Nadel, 1978; Suzuki et al., 1997; Gaffan, 1998; Wiebe and Stäubli, 1999; Moita et al., 2003).

\section{References}

Alvarado MC, Bachevalier J (2005) Comparison of the effects of damage to the perirhinal and parahippocampal cortex on transverse patterning and location memory in rhesus macaques. J Neurosci 25:1599-1609.

Blair (1996) Simulation of a thalamocortical circuit for computing directional heading in the rat. In: Advances in neural information processing systems 8 (Touretzky DS, Mozer MC, Hasselmo ME, eds), pp 152-158. Cambridge, MA: MIT.

Blair HT, Sharp PE (1996) Visual and vestibular influences on head direction cells in the anterior thalamus of the rat. Behav Neurosci 110:643-660.

Brown JE, Skaggs WE (2002) Concordant and discordant coding of spatial location in populations of hippocampal CA1 pyramidal cells. J Neurophysiol 88:1605-1613.

Brown MW, Aggleton JP (2001) Recognition memory: what are the roles of the perirhinal cortex and hippocampus? Nat Rev Neurosci 2:51-61.

Brun VH, Otnass MK, Molden S, Steffenach HA, Witter MP, Moser MB, Moser EI (2002) Place cells and place recognition maintained by direct entorhinal-hippocampal circuitry. Science 296:2243-2246.

Buzsaki G (1986) Hippocampal sharp waves: their origin and significance. Brain Res 398:242-252.

Calton JL, Stackman RW, Goodridge JP, Archey WB, Dudchenko PA, Taube JS (2003) Hippocampal place cell instability after lesions of the head direction cell network. J Neurosci 23:9719-9731.

Cressant A, Muller RU, Poucet B (1997) Failure of centrally placed objects to control the firing fields of hippocampal place cells. J Neurosci 17:2531-2542.

Davachi L, Mitchell JP, Wagner AD (2003) Multiple routes to memory: distinct medial temporal lobe processes build item and source memories. Proc Natl Acad Sci USA 100:2157-2162.

Dudchenko PA, Muir GM, Frohardt RJ, Taube JS (2005) What does the head direction cell system actually do? In: Head direction cells and the neural mechanisms of spatial orientation (Wiener SI, Taube JS, eds), pp 221-246. Cambridge, MA: MIT.

Eichenbaum H (2004) Hippocampus: cognitive processes and neural representations that underlie declarative memory. Neuron 44:109-120.

Fenton AA, Csizmadia G, Muller RU (2000) Conjoint control of hippocampal place cell firing by two visual stimuli. I. The effects of moving the stimuli on firing field positions. J Gen Physiol 116:191-209.

Gaffan D (1998) Idiothetic input into object-place configuration as the contribution to memory of the monkey and human hippocampus: a review. Exp Brain Res 123:201-209.

Goodridge JP, Taube JS (1997) Interaction between the postsubiculum and anterior thalamus in the generation of head direction cell activity. J Neurosci 17:9315-9330.

Goodridge JP, Dudchenko PA, Worboys KA, Golob EJ, Taube JS (1998) Cue control and head direction cells. Behav Neurosci 112:749-761.

Guzowski JF, Knierim JJ, Moser EI (2004) Ensemble dynamics of hippocampal regions CA3 and CA1. Neuron 44:581-584.

Hafting T, Fyhn M, Molden S, Moser MB, Moser E (2005) Microstructure of a spatial map in the entorhinal cortex. Nature 436:801-806.

Hargreaves EL, Rao G, Lee I, Knierim JJ (2005) Major dissociation between medial and lateral entorhinal input to dorsal hippocampus. Science 308:1792-1794.

Humphrey DR, Schmidt EM (1990) Extracellular single-unit recording methods In: Neuromethods, Vol 15, Neurophysiological techniques: applications to neural systems (Boulton AA, Baker GB, Vanderwolf $\mathrm{CH}$, eds), pp 1-54. Clifton, NJ: Humana.

Jeffery KJ, O’Keefe JM (1999) Learned interaction of visual and idiothetic cues in the control of place field orientation. Exp Brain Res 127:151-161.

Knierim JJ (2002a) Dynamic interactions between local surface cues, distal landmarks, and intrinsic circuitry in hippocampal place cells. J Neurosci 22:6254-6264.

Knierim JJ (2002b) The path-integration properties of hippocampal place cells. In: The neural basis of navigation: evidence from single cell recording (Sharp PE, ed), pp 41-58. New York: Kluwer. 
Knierim JJ (2005) Coupling between head direction cells and place cells: Influences of landmarks, self-motion, an intrinsic circuitry. In: Head direction cells and the neural mechanisms of spatial orientation (Wiener SI, Taube JS, eds), pp 163-185. Cambridge, MA: MIT.

Knierim JJ, Kudrimoti HS, McNaughton BL (1995) Place cells, head direction cells, and the learning of landmark stability. J Neurosci 15:1648-1659.

Knierim JJ, Kudrimoti HS, McNaughton BL (1998) Interactions between idiothetic cues and external landmarks in the control of place cells and head direction cells. J Neurophysiol 80:425-446.

Lee I, Yoganarasimha D, Rao G, Knierim JJ (2004) Comparison of population coherence of place cells in hippocampal subfields CA1 and CA3. Nature 430:456-459.

Lindquist DH, Jarrard LE, Brown TH (2004) Perirhinal cortex supports delay fear conditioning to rat ultrasonic social signals. J Neurosci 24:3610-3617.

McFarland NR, Haber SN (2002) Thalamic relay nuclei of the basal ganglia form both reciprocal and nonreciprocal cortical connections, linking multiple frontal cortical areas. J Neurosci 22:8117-8132.

McNaughton BL, Barnes CA, Gerrard JL, Gothard K, Jung MW, Knierim JJ, Kudrimoti H, Qin Y, Skaggs WE, Suster M, Weaver KL (1996) Deciphering the hippocampal polyglot: the hippocampus as a path integration system. J Exp Biol 199:173-185.

Mizumori SJ, Williams JD (1993) Directionally selective mnemonic properties of neurons in the lateral dorsal nucleus of the thalamus of rats. J Neurosci 13:4015-4028.

Moita MA, Rosis S, Zhou Y, LeDoux JE, Blair HT (2003) Hippocampal place cells acquire location-specific responses to the conditioned stimulus during auditory fear conditioning. Neuron 37:485-497.

Muller RU, Kubie JL (1987) The effects of changes in the environment on the spatial firing of hippocampal complex spike cells. J Neurosci 7:1951-1968.

Muller RU, Kubie JL, Ranck Jr JB (1987) Spatial firing patterns of hippocampal complex-spike cells in a fixed environment. J Neurosci 7:1935-1950.

Muller RU, Ranck Jr JB, Taube JS (1996) Head direction cells: properties and functional significance. Curr Opin Neurobiol 6:196-206.

Norman G, Eacott MJ (2005) Dissociable effects of lesions to the perirhinal cortex and the postrhinal cortex on memory for context and objects in rats. Behav Neurosci 119:557-566.

O'Keefe J, Burgess N (1996) Geometric determinants of the place fields of hippocampal neurons. Nature 381:425-428.

O'Keefe J, Burgess N (2005) Dual phase and rate coding in hippocampal place cells: theoretical significance and relationship to entorhinal grid cells. Hippocampus 15:853-866.

O'Keefe J, Conway DH (1978) Hippocampal place units in the freely moving rat: why they fire where they fire. Exp Brain Res 31:573-590.

O'Keefe J, Dostrovsky J (1971) The hippocampus as a spatial map: preliminary evidence from unit activity in the freely moving rat. Brain Res 34:171-175.

O'Keefe J, Nadel L (1978) The hippocampus as a cognitive map. Oxford: Clarendon.

Ragozzino KE, Leutgeb S, Mizumori SJY (2001) Dorsal striatal head direction and hippocampal place representations during spatial navigation. Exp Brain Res 139:372-376.

Ranck Jr JB (1985) Head direction cells in the deep cell layer of dorsal presubiculum in freely moving rats. In: Electrical activity of archicortex (Buzsaki G, Vanderwolf CH, eds), pp 217-220. Budapest: Akademiai Kiado.

Redish AD, Elga AN, Touretzky DS (1996) A coupled attractor model of the rodent head direction system. Network 7:671-685.

Save E, Poucet B (2000) Involvement of the hippocampus and associative parietal cortex in the use of proximal and distal landmarks for navigation. Behav Brain Res 109:195-206.

Shapiro ML, Tanila H, Eichenbaum H (1997) Cues that hippocampal place cells encode: dynamic and hierarchical representation of local and distal stimuli. Hippocampus 7:624-642.

Sharp PE, Blair HT, Etkin D, Tzanetos DB (1995) Influences of vestibular and visual motion information on the spatial firing patterns of hippocampal place cells. J Neurosci 15:173-189.

Sharp PE, Blair HT, Cho J (2001) The anatomical and computational basis of the rat head-direction cell signal. Trends Neurosci 24:289-294.

Skaggs WE, McNaughton BL, Gothard KM, Markus EJ (1993) An information-theoretic approach to deciphering the hippocampal code. In: Advances in neural information processing systems (Hanson SJ, Cowan JD, Giles CL, eds), pp 1030-1037. San Mateo, CA: Kaufman.

Skaggs WE, Knierim JJ, Kudrimoti HS, McNaughton BL (1995) A model of the neural basis of the rat's sense of direction. Adv Neural Inf Process Syst 7:173-180.

Skaggs WE, McNaughton BL, Wilson MA, Barnes CA (1996) Theta phase precession in hippocampal neuronal populations and the compression of temporal sequences. Hippocampus 6:149-172.

Song P, Wang X-J (2005) Angular path integration by moving "hill of activity": A spiking neuron model without recurrent excitation of the headdirection system. J Neurosci 25:1002-1014.

Stackman RW, Clark AS, Taube JS (2002) Hippocampal spatial representations require vestibular input. Hippocampus 12:291-303.

Stackman RW, Golob EJ, Bassett JP, Taube JS (2003) Passive transport disrupts directional path integration by rat head direction cells. J Neurophysiol 90:2862-2874.

Stringer SM, Rolls ET, Trappenberg TP (2004) Self-organising continuous attractor networks with multiple activity packets, and the representation of space. Neural Netw 17:5-27.

Suzuki WA, Miller EK, Desimone R (1997) Object and place memory in the macaque entorhinal cortex. J Neurophysiol 78:1062-1081.

Tanila H, Shapiro ML, Eichenbaum H (1997) Discordance of spatial representation in ensembles of hippocampal place cells. Hippocampus 7:613-623.

Taube JS (1995) Head direction cells recorded in the anterior thalamic nuclei of freely moving rats. J Neurosci 15:70-86.

Taube JS (1998) Head direction cells and the neurophysiological basis for a sense of direction. Prog Neurobiol 55:225-256.

Taube JS, Burton HL (1995) Head direction cell activity monitored in a novel environment and during a cue conflict situation. J Neurophysiol 74:1953-1971.

Taube JS, Muller RU, Ranck Jr JB (1990a) Head-direction cells recorded from the postsubiculum in freely moving rats. I. Description and quantitative analysis. J Neurosci 10:420-435.

Taube JS, Muller RU, Ranck Jr JB (1990b) Head-direction cells recorded from the postsubiculum in freely moving rats. II. Effects of environmental manipulations. J Neurosci 10:436-447.

Tulving E, Markowitsch HJ (1998) Episodic and declarative memory: role of the hippocampus. Hippocampus 8:198-204.

Vargha-Khadem F, Gadian DG, Watkins KE, Connelly A, Van Paesschen W, Mishkin M (1997) Differential effects of early hippocampal pathology on episodic and semantic memory. Science 277:376-380.

Wiebe SP, Stäubli UV (1999) Dynamic filtering of recognition memory codes in the hippocampus. J Neurosci 19:10562-10574.

Wiener SI (1993) Spatial and behavioral correlates of striatal neurons in rats performing a self-initiated navigation task. J Neurosci 13:3802-3817.

Wiener SI, Korshunov VA, Garcia R, Berthoz A (1995) Inertial, substratal and landmark cue control of hippocampal CA1 place cell activity. Eur J Neurosci 7:2206-2219.

Wiener SI, Berthoz A, Zugaro MB (2002) Multisensory processing in the elaboration of place and head direction responses by limbic system neurons. Brain Res Cogn Brain Res 14:75-90.

Yoganarasimha D, Knierim JJ (2005) Coupling between place cells and head direction cells during relative translations and rotations of distal landmarks. Exp Brain Res 160:344-359.

Yoganarasimha D, Hargreaves EL, Knierim JJ (2003) Rotational coherence of head direction, medial entorhinal, and CA1 place cell ensembles. Soc Neurosci Abstr 29:91.12

Young BJ, Fox GD, Eichenbaum H (1994) Correlates of hippocampal complex-spike cell activity in rats performing a nonspatial radial maze task. J Neurosci 14:6553-6563.

Zar JH (1999) Biostatistical analysis. Upper Saddle River, NJ: Prentice Hall.

Zhang K (1996) Representation of spatial orientation by the intrinsic dynamics of the head-direction cell ensembles: a theory. J Neurosci 16:2112-2126.

Zinyuk L, Kubik S, Kaminsky Y, Fenton AA, Bures J (2000) Understanding hippocampal activity by using purposeful behavior: place navigation induces place cell discharge in both task-relevant and task-irrelevant spatial reference frames. Proc Natl Acad Sci USA 97:3771-3776.

Zugaro MB, Berthoz A, Wiener SI (2001) Background, but not foreground, spatial cues are taken as references for head direction responses by rat anterodorsal thalamus neurons. J Neurosci 21:RC154(1-5).

Zugaro MB, Arleo A, Berthoz A, Wiener SI (2003) Rapid spatial reorientation and head direction cells. J Neurosci 23:3478-3482.

Zugaro MB, Arleo A, Dejean C, Burguiere E, Khamassi M, Wiener SI (2004) Rat anterodorsal thalamic head direction neurons depend upon dynamic visual signals to select anchoring landmark cues. Eur J Neurosci 20:530-536. 